\title{
ABSTRACTS
}

\section{9th annual meeting of the scientific association of swiss radiation oncology (SASRO)}

\author{
11.-13. June 2015 \\ Basel
}

\section{Organizing committees}

\section{Congress president}

Frank Zimmermann

Scientific committee in radiation oncology - clinicians

Daniel Aebersold

Abdelkarim Allal

Karl Beer

Stephan Bodis

Jean Bourhis

Alan Dal Pra

Alessandra Franzetti Pellanda

Gross Markus

Guenther Gruber

Matthias Guckenberger

Urs Meier

Raymond Miralbell

René Mirimanoff

Markus Notter

Alexandros Papachristofilou

Gianfranco Pesce

Ludwig Plasswilm

Antonella Richetti

Peter Thum

Damien Charles Weber

Daniel Zwahlen

Scientific committee in radiation oncology-medical physicists

Maria Mania Aspradakis

Stephan Klöck

\author{
Götz Kohler \\ Tony Lomax \\ Gerd Lutters \\ Raphaël Moeckli \\ Peter Pemler \\ Daniel Vetterli
}

Scientific committee in radiation

oncology_biologists

Gianfranco Pesce

Martin Pruschy

Jitzhak Zimmer

Kathrin Zaugg

Scientific committee in radiation oncology-technicians

Bert Pastoors

Dechantsreiter Ulrike

Krummenacher Sabine

Renate Nyffenegger

Scientific committee in radiation oncology—nurses Dubravka Miletic

Susanne Sester

Scientific committee in radiation oncologysecretaries and medical practice assistants

Claude Breitenstein

Silvana Rossi

Claire Solinger

Monika Strohmeier

\section{Abstracts overview}

Biology

Clinics

Physics 


\section{Biology}

Bio-01

Impact of MET inhibition on gene expression mediated by HIF$1 \alpha$ signaling in tumor cells under hypoxic conditions

A. Glück ${ }^{1,2}$, L. Nisa ${ }^{1,2}$, M. Medová ${ }^{1,2}$, D.M. Aebersold ${ }^{1,2}$, Y. Zimmer,

${ }^{1}$ Department of Radiation Oncology, Inselspital, University of Bern, Bern, Switzerland; ${ }^{2}$ Department of Clinical Research, Radiation Oncology, University of Bern, Bern, Switzerland

Aims Hypoxia is a fundamental biologic feature largely involved in aggressive manifestations of tumor growth and progression. Previous studies showed that hypoxic areas of tumors overexpress the MET tyrosine kinase, a high affinity receptor for hepatocyte growth factor (HGF). As both MET and hypoxia are determinants that affect cellular responses to DNA damaging agents, we aimed in this study to elucidate the effect of MET inhibition (METi) on MET-expressing tumor cell lines under hypoxic conditions.

Methods MET-overexpressing cancer cell lines were exposed to a hypoxic environment $\left(1.5 \% \mathrm{O}_{2}\right)$. METi was obtained using different pharmacological inhibitors. Transcription of genes of interest was assessed by qRT-PCR, protein expression by western blotting and apoptosis by Caspase- 3 enzymatic activity assays. The hypoxic transcription factor HIF-1 $\alpha$ was stabilized through the use of dimethyloxaloylglycine or MG-132.

Results Following METi, protein levels of HIF- $1 \alpha$ and its targets are decreased. Transcription of HIF-1 $\alpha$ is not affected as seen from its stable mRNA levels. Despite prolyl-hydroxylase (a key enzyme responsible for HIF-1 $\alpha$ degradation) or proteasome inhibition, HIF-1 $\alpha$ protein levels remain reduced upon METi, suggesting potentially reduced HIF- $1 \alpha$ translation.

Conclusion We have identified a link between MET and HIF- $1 \alpha$ signaling which is likely to be important in tumor progression. Interruption of this pathway using MET inhibitors may contribute to antitumor activity of these agents in hypoxic malignancies. In future, it will be of great interest to investigate whether targeting cells with MET inhibitors followed by ionizing radiation delays growth and reduces survival of hypoxic tumor cells.

\section{Bio-02}

\section{Targeting the MET receptor tyrosine kinase as a strategy for} radiosensitization in head and neck cancer preclinical models

L. Nisa ${ }^{1,2,3}$, M. Medová1,2, R. Giger ${ }^{3}$, M. D. Caversaccio ${ }^{3}$, A. Quintin ${ }^{1,2}$, D.M. Aebersold ${ }^{1,2}$, Y. Zimmer $^{1,2}$

${ }^{1}$ Department of Radiation Oncology, Inselspital, Bern University Hospital and University of Bern, 3010 Bern, Switzerland; ${ }^{2}$ Department of Clinical Research, Inselspital, Bern University Hospital and University of Bern, 3010 Bern, Switzerland; ${ }^{3}$ Department of Otorhinolaryngology - Head and Neck Surgery, Inselspital, Bern University Hospital and University of Bern, 3010 Bern, Switzerland

Aims The goal of this study is to assess the impact of MET inhibition in combination with ionizing radiation (IR) in Head and Neck Cancer (HNC) preclinical models.

Material and methods We used a panel of HNC cell lines to assess levels of MET and active pTyr-1234/5-MET (pMET) expression, cell proliferation, clonogenic survival, migration, and induction of apoptosis in response to MET inhibition combined with IR. MET inhibition (METi) was performed using a novel and specific ATP-competitive tyrosine kinase inhibitor.
Additionally, patient-derived organotypic tissue slice cultures (OTSCs) were generated from fresh resection specimens using a Vibratome VT1200S. OTSCs measuring $350 \mu \mathrm{m}$ were exposed to METi, IR, or combinations of both modalities. Endpoints included immunohistochemical levels of MET and pMET, as well as markers of proliferation (Ki67) and apoptosis (cleaved caspase-3).

Results Cell lines and OTSCs usually express high levels of total MET, and variable levels of pMET. Upon METi, downstream effects are variable. In cell lines METi in combination with IR elicits differential responses, but a decrease in clonogenic survival and cell migration, as well as induction of caspase-3 activity are usually seen in a METi and IR dose-dependent manner.

OTSCs equally display differential responses to METi and IR, and indicate the potential of METi to sensitize radiation unresponsive MET-expressing tumors to IR.

Conclusion MET targeted inhibition is a promising strategy for radiosensitization in HNC preclinical models. The differential responses observed in patient-derived OTSCs strongly indicate the need for proper pre-therapeutic stratification to select patients who will likely benefit from such combination regime. Additional approaches will be conducted to elucidate underlying mechanisms of MET inhibition in OTSCs.

\section{Clinics}

\section{Clin-01}

Title: Neutrophil-to-Lymphocyte Ratio Predicts Overall Survival following Chemoradiation for Locally Advanced Head and Neck Cancers

B. Bojaxhiu M.D. ${ }^{1}$, A.J. Templeton M.D. ${ }^{2}$, O. Elicin M.D. ${ }^{1}$, J.O Brömme M.D. ${ }^{1}$, K. Zaugg M.D. ${ }^{1}$, R. Giger M.D. ${ }^{3}$, D.M Aebersold M.D. ${ }^{1}$, A. Dal Pra M.D. ${ }^{1}$

${ }^{1}$ Department of Radiation Oncology, Inselspital, Bern University Hospital and University of Bern, Bern, Switzerland; ${ }^{2}$ Department of Medical Oncology, Kantonsspital St. Gallen, St. Gallen, Switzerland; ${ }^{3}$ Department of Otorhinolaryngology, Bern University Hospital, Bern, Switzerland

Purpose A high neutrophil-to-lymphocyte ratio (NLR) is a marker of systemic inflammation reported to be a poor prognostic factor in many diseases. This study aimed at investigating the prognostic value of NLR in patients with head and neck squamous cell carcinoma (HNSCC) treated with chemoradiation.

Methods A cohort of locally advanced HNSCC patients treated with curative chemoradiation between 2007 and 2010 was analyzed. NLR was computed using pre-treatment complete blood counts performed within 10 days of treatment start. The prognostic role of NLR was explored with univariate and multivariate Cox regression analyses with adjustment for disease-specific prognostic factors. NLR was assessed as a log-transformed continuous variable ( $\operatorname{logNLR}$ ) and dichotomized by the optimal cut-off value based on the highest area under the receiver operating characteristic (ROC) curve.

Results The cohort included 186 patients, 52 (28\%) with oral cavity, $83(45 \%)$ with oropharyngeal, $27(14 \%)$ with hypopharyngeal and 24 (13\%) with laryngeal cancers. Median follow up was 50 months. A NLR of 4 was determined as the optimal cutoff value. On univariate analysis, age $(P=0.02)$, tumor location $(P=0.03)$, AJCC stage $(P=0.03)$ and NLR (logNLR, $P=0.01$; NLR $>4, P=0.01)$ were associated with overall survival (OS). NLR was not associated with locoregional or distant recurrences. On multivariate analysis, Karnofsky Performance Status, tumor location, and higher NLR were associated with worse OS [logNLR, HR 1.59 (95\% CI 1.01-3.43, $P=0.04)$; NLR $>4$, HR $1.80(95 \%$ CI $1.04-3.13, P=0.03)]$. 
Conclusion Our data shows that NLR is an independent predictor of mortality in HNSCC patients treated with chemoradiation. NLR is a readily available biomarker that could improve pre-treatment prognostication and potentially enhance patient stratification in clinical studies.

\section{Clin-02}

Gross Tumor Volume (GTV) Evolution of Lung Lesions Throughout Stereotactic Body Radiation Therapy (SBRT) and at first post-treatment control

T. Breuneval M.D., P. Tsoutsou M.D., K. Khanfir M.D., C. Castella Ph.D.

Department of Radiation Oncology, Hôpital du Valais, Sion, Switzerland

Aims The goal of this study was to evaluate the GTV variation of lung lesions throughout a SBRT treatment and at first post-treatment control.

Methods Between May 2014 and April 2015, nine inoperable patients diagnosed with non-small cell lung cancer (NSCLC) of stage up to IB (one pt without histology) were treated with SBRT $(5 \times 12$ Gy or $8 \times 6-7.5$ Gy). All patients underwent 4D- computed tomography (CT) scan for planning and Cone Beam CT (CBCT) for verification before each fraction. A post-treatment diagnostic $\mathrm{CT}$ was undertaken at 8-10 weeks. All GTVs from each phase of the 4D-planning CT and from each first CBCT of every treatment fraction were contoured. Similarly, the post-treatment tumor volume in the $\mathrm{CT}$ at $8-10$ weeks and the pretreatment one were contoured and compared. The volume variation was analyzed using 1-way analysis of variance (ANOVA) and t-tests.

Results When normalized to the first treatment day, a significant difference in the GTV relative volume is demonstrated for subsequent fractions (ANOVA, $p=0.001$ ) (Fig. 1). While inflammation causes the mean relative volume of the GTV across patients to initially expand, it is $20 \%$ smaller at the end of treatment ( $p<10^{-4}$ at fraction 5, $p=0.04$ at fraction 8 ). Furthermore, the post-treatment control CT confirms the shrinkage of the GTV on average by a five-fold factor (range: 1.8-8.3) compared to the planning CT $(p<0.01)$.

Conclusion GTV evolution throughout and after SBRT in patients with NSCLC is captured on CTs; it follows a pattern consistent across all patients and possibly provides information about inflammation and short-term post-treatment response.

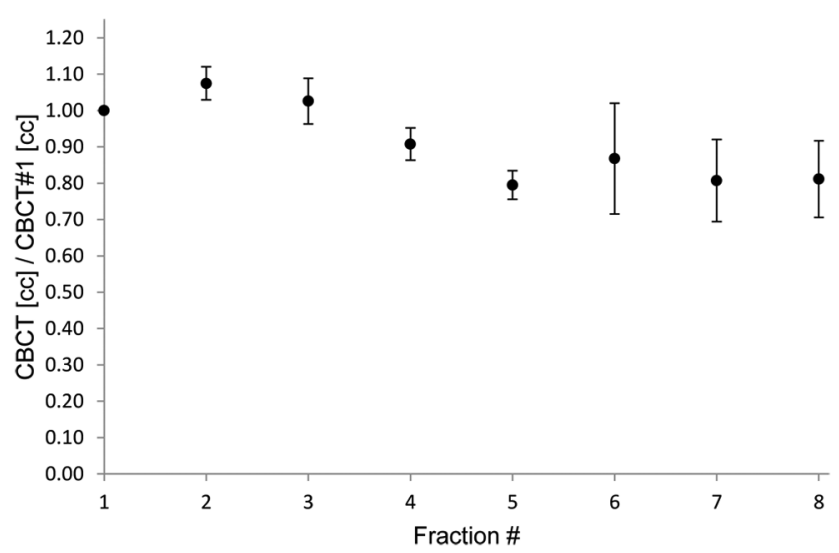

Fig. 1 Clin-02. Evolution of the GVT relative volume throughout SBRT. The results are averaged across all patients. Error bars represent the standard error of the mean
Clin-03

\section{Deep inspiration breath hold with RapidArc in bilateral breast adjuvant radiotherapy}

S. Cima ${ }^{1}$, M. Frapolli ${ }^{1}$, G. Nicolini ${ }^{2}$, K. Yordanov ${ }^{1}$, G. Pesce ${ }^{1}$ C. Azinwi ${ }^{1}$, F. Martucci ${ }^{1}$, J.J Stelmes ${ }^{1}$, A. Richetti ${ }^{1}$, M.C. Valli ${ }^{1}$

'Radiation Oncology, Oncology Institute of Southern Switzerland, Bellinzona-Lugano, CH, Switzerland; ${ }^{2}$ Medical Physics Service, Ente Ospedaliero Cantonale, Bellinzona, $\mathrm{CH}$, Switzerland

Objective To evaluate the feasibility and the impact of deep inspiration breath hold (DIBH) in bilateral breast cancer adjuvant radiotherapy treated with RapidArc technique.

Material and methods We analyzed fourteen female patients diagnosed with synchronous bilateral breast cancer, treated with conservative surgery and adjuvant radiotherapy from December 2008 to April 2014. All patients were irradiated on both breasts plus simultaneous integrated bilateral boost using RapidArc technique.

Results Prescription to the target mean dose ranged from 50 to 50.4 Gy for the whole breast and from 62.5 to 67.2 Gy for the boost volume. The patients have been treated 5 days per week ending the whole treatment in nearly 6 weeks. Six patients $(43 \%)$ have been treated with DIBH, starting from 2011.

The average mean dose to the heart was 6.0 Gy in case of DIBH (3.1-9.5 Gy) and 8.9 Gy (6.9-12.2 Gy) in free breathing condition $(p=0.02)$.

The average mean dose given to the left anterior descending artery (LAD) was $14.39 \mathrm{~Gy}(4.8-23.7 \mathrm{~Gy})$ in case of DIBH and 25.1 Gy (15.8-29.4 Gy) without DIBH ( $p=0.01)$.

DVHs for both lungs showed an average V20 Gy of $10.4 \%$ (8.9$15.0 \%)$ and $10.7 \%(5.0-17.1)$ respectively $(p=0.48)$.

Conclusion Bilateral breast radiotherapy delivered with RapidArc and DIBH is feasible. All patients selected for DIBH smoothly concluded their treatment, without compromising the session treatment time. DIBH allowed a better spare of the LAD and heart, suggesting its routine use in this setting of patients

\section{Clin-04}

A systematic review and meta-analysis for re-irradiation with hyperthermia for loco-regional recurrent breast cancers

N. R. Datta ${ }^{1}$, E. Puric ${ }^{1}$, D. Klingbiel ${ }^{2}$, S. Bodis ${ }^{1,3}$

${ }^{1}$ Centre for Radiation Oncology KSA-KSB, Kantonsspital Aarau, Aarau, Switzerland; ${ }^{2}$ Department of Statistics, Swiss Group of Clinical Cancer Research (SAKK), Coordinating Centre, Bern, Switzerland; ${ }^{3}$ Department of Radiation Oncology, University Hospital Zurich, Zurich, Switzerland

Aims Local hyperthermia (HT) along with moderate doses of re-irradiation (ReRT) has been used in various single-arm (ReRT + HT) and two-arm clinical trials (randomized and nonrandomized, ReRT vs. $\mathrm{ReRT}+\mathrm{HT}$ ) to treat loco-regional recurrent breast cancers (LRBCs). We conduct a systematic review and meta-analysis on the outcome of these trials.

Material/methods A detailed search was conducted as per the PRISMA guidelines. 2047 abstracts were screened from the 10 databases. Only those studies which had received prior loco-regional RT and were treated with ReRT + HT alone were considered. End point evaluation was complete loco-regional response (CR) following ReRT + HT.

Results A total of 43 full papers were shortlisted and reviewed, and 24 studies were selected for detailed review based on pre-defined selection criteria. Six of these were two-arm (randomized, $n=4$; nonrandomized, $n=2$ ) while 18 were single-arm studies resulting in a total of 1319 patients of which 1162 received ReRT + HT. Two-arm stud- 
ies included 494 patients, and a CR of 62.8 and $39.3 \%$ was observed with ReRT + HT and ReRT respectively, giving an odds ratio of 2.9 (95\%CI: $\left.1.8-4.6, p<0.001 ; \mathrm{I}^{2}=22.2, p=0.26\right)$. The risk ratio was 1.5 (95\%CI: 1.2-2.0) in favor of ReRT + HT. For 18 single-arm studies, a CR of $64.6 \%$ was reported from the 825 patients resulting in an event rate of 0.6 (95\%CI: $\left.0.5-0.6, p<0.001 ; \mathrm{I}^{2}=49.7, p=0.008\right)$. The risk difference was $23.9 \%$ favouring ReRT + HT over ReRT alone $(p<0.001)$. Mean acute and late grade III/IV toxicities with ReRT + HT were reported as 13.4 and $3.9 \%$.

Conclusions Moderate dose of ReRT $+\mathrm{HT}$ enhances the $\mathrm{CR}$ rates by 1.5 times to that of ReRT alone in pre-irradiated LRBCs with low acute and late morbidities and thus could be considered an effective modality to treat such patients.

\section{Clin-05}

\section{Radiotherapy infrastructure and human resources in} Switzerland: Present status and projections for 2020

\section{N. R. Datta ${ }^{1}$, S. Bodis ${ }^{1,2}$}

${ }^{1}$ RadioOnkologieZentrum, KSA-KSB, Kantonsspital Aarau, Aarau, Switzerland; ${ }^{2}$ Department of Radiation Oncology, University Hospital Zurich, Zurich, Switzerland

Aims The present status and requirements by 2020 of radiotherapy (RT) infrastructure and staffing in Switzerland, in terms of teletherapy (TRT) units, radiation oncologists (ROs), medical physicists (MPs) and RT technologists (RTTs) are presented.

Methods Site specific cancer incidences for each of the 24 commonest cancers in Switzerland were mined from the GLOBOCAN 2012 database and estimated for 2015 and 2020. RT capacity was obtained from the Directory of Radiotherapy Centres (DIRAC), IAEA. Total patients requiring RT in 2015 and 2020 were computed using the RT utilization rates (RTU) for each cancer site (1). The IAEA and ESTROQUARTS guidelines were used to estimate RT infrastructure and personnel.

Results Switzerland, presently is one of the few European countries with a $100 \%$ RT access to 24,853 patients (2). The available TRTs (65), ROs (97) and MPs (55) are adequate and comply with the recommended norms. However, an additional 51 RTTs (presently 114) are needed to meet the current requirement of $1 / 150$ patients per year. By 2020 , RT would be required in 27,289 patients, resulting in an additional need of 3, 12, 5 and 68 of TRTs, ROs, MPs and RTTs respectively.

Conclusions Switzerland presently has an acceptable RT infrastructure and staffing, except for RTTs. Efforts are needed to bridge this gap to further optimize the RT workflow and patient care. With changing RT practice, the RTU could change over the next 5 years which needs to be accounted for future planning. Certain pragmatic steps would be discussed.

\section{References}

1. Barton M, et al. (2013) Review of optimal radiotherapy utilisation rates (On internet)

2. Datta NR, et al. (2014) Radiotherapy infrastructure and human resources in Europe - present status and its implications for 2020 . Europ J Cancer, 2735-43

\section{Clin-06}

\section{Outcome of Proximal Esophageal Cancer After Definitive Combined Chemo-Radiation: A Multicenter Swiss Retrospective Study}

E. Herrmann ${ }^{1}$, N. Mertineit ${ }^{1}$, B. De-Bari ${ }^{2}$, L. Hoeng ${ }^{3}$, F. Caparrotti ${ }^{4}$, R. Jumeau ${ }^{2}$, O. Elicin ${ }^{1}$, N. Cihoric ${ }^{1}$, P. Wolfensberger ${ }^{1}$, T. Lössl ${ }^{1}$, D.M. Aebersold ${ }^{1}$, M. Ozsahin ${ }^{2}$

${ }^{1}$ Department of Radiation Oncology, Bern University Hospital, and University of Bern, Bern, Switzerland; ${ }^{2}$ Department of Radiation Oncology, Lausanne University Hospital, Lausanne, Switzerland; ${ }^{3}$ Department of Radiation Oncology, Kantonsspital St. Gallen, St. Gallen, Switzerland; ${ }^{4}$ Radiation Oncology Department, Geneva University Hospital, Geneva, Switzerland

Objective To report oncological outcome and toxicity of definitive external beam radiotherapy (RT) combined with platin-based chemotherapy (CRT) in the treatment of proximal esophageal cancer.

Methods The medical records of patients with stage cT1-4 cN0-3 cM0 proximal esophageal cancer treated with platin-based definitive CRT between 2004-2013 in four Swiss institutions were retrospectively studied. Acute and chronic toxicities were evaluated with CTCAE v.4. The impact of possible patient related and procedural characteristics on loco-regional control (LRC), overall survival (OS) and disease-free survival (DFS) were evaluated using the log-rank test and multivariate Cox proportional hazards model. Primary endpoint was LRC.

Results We included 55 patients. Mean age was 65 (range: 42-78) with a median follow up of 34 months (range: 6-110). Median time interval from diagnosis to RT was 89 days (range: 6-178). Median radiation dose was 56 Gy (range 28-72). Induction chemotherapy (ICHT) was given in $58 \%$ of patients. Actuarial LRC, DFS and OS at 3 years were $52 \%(95 \%$ CI: $37-67 \%), 35 \%$ (95\% CI: $22-50 \%)$ and $52 \%$ (95\% CI: 37-67\%), respectively. On univariate analyses, the only significant predictive factor for LRC was the time interval from diagnosis to RT. During RT, acute toxicities (dysphagia, pain, skinreaction) ranged from grade 0 to 3 , without significant dose-dependent differences. Dysphagia at last follow-up ranged from grade 0 to 4 . After backwards elimination, the multivariate model showed a total radiation dose $>56$ Gy together with ICHT as predictive factors for DFS and OS $(p<0.05)$.

Conclusion Combined CRT seems to be a reliable therapeutic option for proximal esophageal cancer. Randomized prospective trials are needed to optimize this combined treatment modality for increased DFS and OS.

\section{Clin-07}

Interest and feasibility of integration of ${ }^{99} \mathrm{mTc}$-mebrofenin SPECT into a stereotactic radiotherapy plan for liver cancer

\section{R. Jumeau ${ }^{1}$, M. Ozsahin ${ }^{1}$, J. Prior ${ }^{2}$, J. Bourhis ${ }^{1}$, B. De Bari ${ }^{1}$}

${ }^{1}$ Department of radio-oncology, Lausanne university hospital, Suisse, Lausanne, Switzerland ${ }^{2}$ Department of nuclear medicine, Lausanne university hospital, Suisse, Lausanne, Switzerland

Introduction Stereotactic body radiotherapy (SBRT) is an alternative to surgery in treatment of liver cancers (LC) or metastases. ${ }^{99} \mathrm{mTc}$ mebrofenin single-photon emission computed tomography $\left({ }^{99} \mathrm{TcM}-\right.$ SPECT) is usually used to determine the function of remnant liver after surgery. In our original approach, we integrated data of ${ }^{99} \mathrm{TcM}$ SPECT into a SBRT plan for LC to spare the most functional areas. Material and methods A 84-year-old patient suffering from cirrhosis presented a LC in the right liver. Due to comorbidity, surgery was not possible and SBRT treatment was decided. We performed a planning computed tomography for radiotherapy (RTCT) and a few days after 
a ${ }^{99} \mathrm{TcM}-\mathrm{SPECT}$ that we registered with the RTCT. Structures corresponding to the liver function (LF) were created and constraints were applied on most functioning areas. Three months after treatment we performed another ${ }^{99} \mathrm{TcM}$-SPECT. To understand the effects of SBRT on LF, we compared the variation of LF structures before and after treatment.

Results We delivered a total dose of 45 Gy in 3 fractions using volumetric modulated arc therapy without any complication. All of LF structures were smaller after treatment than before (Fig. 1). We noticed more changes at the same regimen of dose for well functioning liver. The average variation of poorly functioning liver $(0-30 \%$ LF) was $46 \%$ (range, 16-60\%), and was $80 \%$ (range, 52-87\%) for the well functioning liver $(40-60 \%$ LF). This difference was statistically significant $(p<0.01)$.

Conclusion Integration of ${ }^{99} \mathrm{TcM}-\mathrm{SPECT}$ into RT plans is possible and useful to spare the most functional liver areas. The interest is to protect the well functioning liver that seems to be more sensitive to SBRT.

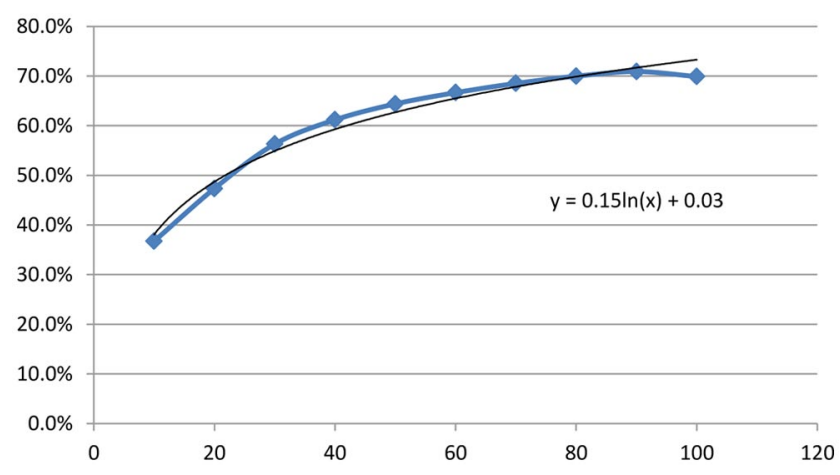

Fig. 1 Clin-07. Variation of liver function according to the dose (expressed as equivalent dose in 2 Gy per fraction)

\section{Clin-08}

\section{Short vs protracted urethra-sparing prostate SBRT: feasibility and early toxicity from a randomized phase II trial}

M. Kountouri M.D. ${ }^{1}$, T. Zilli M.D. ${ }^{1}$, A. Bruynzeel M.D. ${ }^{4}$, H. Minn M.D. ${ }^{6}$, E. Sánchez-Saugar M.D. ${ }^{7}$, A. Oliveira M.D. ${ }^{5}$, S. Bral M.D. ${ }^{9}$, S. Jorcano M.D. ${ }^{8}$, U. Abacioglu M.D. ${ }^{3}$, Z. Symon M.D. ${ }^{2}$, R. Miralbell M.D. ${ }^{1,8}$

${ }^{1}$ Geneva University Hospital, Geneva, Switzerland; ${ }^{2}$ Sheba Medical Center, Ramat Gan, Israel; ${ }^{3}$ Neolife Medical Center, Beşiktaş/ İstanbul—Avrupa, Turkey; ${ }^{4} \mathrm{VU}$ University Medical Center, Amsterdam, the Netherlands; ${ }^{5}$ Portuguese Institut of Oncology, Lisbon, Portugal; ${ }^{6}$ University Hospital Turku, Turku, Finland; ${ }^{7}$ Hospital Universitario Sanchinarro, Madrid, Spain; ${ }^{8}$ Teknon Oncologic Institute, Barcelona, Spain; ${ }^{9}$ Onze-Lieve-Vrouwziekenhuis, Aalst/Asse, Belgium

Purpose To evaluate the feasibility and preliminary toxicity results of a prospective randomized multicenter phase II trial of short vs. protracted urethra-sparing SBRT for localized prostate cancer.

Methods A total of $93 \mathrm{cT} 1-3 \mathrm{a}$ N0 M0 prostate cancer patients with a lymph-node involvement risk $\leq 20 \%$ were randomized between 09/2012 and 10/2014 to be treated with an SBRT protocol of $36.25 \mathrm{~Gy}$ in 5 fractions of 7.25 Gy either over 9 days (Arm A, $n=45$ ) or over 28 days once-a-week, (Arm B, $n=48$ ). The dose to the urethra with a $3 \mathrm{~mm}$ margin was $32.5 \mathrm{~Gy}$ in 5 fractions. Tolerance to the treatment was scored using the CTCAE ver. 4.0 grading scale, the IPSS and the EORTC QLQ-PR25 QoL questionnaire.

Results SBRT was delivered to all patients as planned with no treatment interruptions. Overall genitourinary and gastrointestinal toxici- ties were below the stopping rule established for the study, with grade 2 toxicity reported in 6 patients for each arm mostly after the 5th fraction. Only one patient experienced late grade 3 rectal toxicity. Mean IPSS scores increased significantly between baseline and the 5 th fraction $(p<0.01)$ in both treatment arms, returning to baseline at week 12. Mean IPSS values at baseline, after the 5 th fraction and after 12 weeks were 7.5, 14 and 7.5 for Arm A and 8.1, 12.7 and 8.9 for Arm $\mathrm{B}$, respectively. No significant differences were observed in EORTC QLQ-PR25 QoL endpoints between baseline and week 12 in either study arm.

Conclusions Preliminary results demonstrated the feasibility and the acceptable toxicity rates of this short vs. protracted urethra-sparing prostate SBRT phase II trial. Three months after randomization complete recovery from side effects and return to baseline IPSS scores was observed in both treatment schedules.

\section{Clin-09}

\section{Sub-Stratification of Intermediate Risk Group Prostate Cancer by Multi-Parametric MRI Evaluation of $\mathrm{ADC}_{\text {mean }}$ Values and Maximum Tumor Diameter}

N.T. Kummer M.D., Ph.D. ${ }^{1}$, D.J.A. Margolis M.D. ${ }^{2}$, J. Wang Ph.D. ${ }^{1}$, P. Kupelian M.D. ${ }^{1}$, M. Kamrava M.D. ${ }^{1}$

${ }^{1}$ Department of Radiation Oncology, UCLA, Los Angeles, US; ${ }^{2}$ Department of Radiology, UCLA, Los Angeles, US

NCCN defined intermediate risk prostate cancer reflects a heterogeneous population with clinical data demonstrating differing outcomes for Gleason score (GS) 3 versus 4 predominant disease. We investigated if pre-treatment multi-parametric MRI (MP-MRI) evaluation of prostate cancer using maximum tumor diameter (MTD) and mean attenuation diffusion coefficient (ADC) values improve GS 3 vs GS 4 predominant disease stratification. T2 weighted imaging was used to determine maximum tumor diameter (MTD) and diffusion weighted imaging was used to determine an $\mathrm{ADC}_{\text {mean }}$ value, retrospectively in a cohort or 92 patients. GS 3 predominant tumors had a mean MTD of $1.2 \mathrm{~cm}(95 \%$ CI: $1.1-1.3 \mathrm{~cm})$ compared to GS 4 predominant tumors with a mean MTD of $1.8 \mathrm{~cm}(95 \% \mathrm{CI}: 1.5-2.1 \mathrm{~cm} ; p \leq 0.0001)$. AD$\mathrm{C}_{\text {men }}$ values were inversely correlated with bGS $3+3,3+4,4+3$, and Z4+4: $1020 \mathrm{~mm} / \mathrm{s}^{-1}(950-1091), 973 \mathrm{~mm} / \mathrm{s}^{-1}(896-1050), 904 \mathrm{~mm} / \mathrm{s}^{-1}$ (774-1036), and $786 \mathrm{~mm} / \mathrm{s}^{-1}(694-879)\left(\mathrm{R}^{2}=0.15, p<0.002\right)$. ROC analyses resulted in a MTD cut-off value of $1.4 \mathrm{~cm}$ (75\% accuracy, $70 \%$ sensitivity, $77 \%$ specificity, $82 \% \mathrm{NPV}$ ), and $\mathrm{ADC}_{\text {mean }}$ value of $1000 \mathrm{~mm} / \mathrm{s}^{-1}$ (71 \% accuracy, $100 \%$ sensitivity, $56 \%$ specificity, $100 \%$ NPV) for differentiating between bGS 6 and bGS $\geq 8$ tumors. Based on this data an MTD/ADC grouping system from 1 to 4 was developed (1: small MTD, high $\mathrm{ADC}_{\text {mean }}$ to 4: large MTD, low $\mathrm{ADC}_{\text {mean }}$ ). When applied to intermediate risk group patients, they segregated into predominately group 1 and 4 patients (38\% group $1,5 \%$ group $2,15 \%$ group 3 , and $43 \%$ group 4 ). MP-MRI using MTD and $\mathrm{ADC}_{\text {mean }}$ can stratify intermediate risk patients into bGS 3 versus bGS 4 predominant disease. This information may be useful in improving risk stratification for intermediate risk prostate cancer patients.

\section{Clin-10}

DNA Repair Interference with Sequence Specific DNA Binding Pyrrole Imidazole Polyamides; a Novel Radiation Sensitizer

\section{N. Kummer ${ }^{1}$, F. Yang ${ }^{2}$, P. B. Dervan ${ }^{2}$, N. G. Nickols ${ }^{1,2}$}

${ }^{1}$ Department of Radiation Oncology, UCLA, Los Angeles, US; ${ }^{2}$ Division of Chemistry and Chemical Engineering, California Institute of Technology, Pasadena, US 
Pyrrole (Py) - Imidazole ( $\mathrm{Im})$ polyamides are a class of non-genotoxic DNA-binding small molecules with modular sequence recognition and high affinity. A polyamide targeted to the Androgen Receptor (AR) Response Element (ARE) half-site, suppresses AR positive prostate tumor xenografts. It is hypothesized that polyamides may displace DNA repair elements recruited after genotoxic insult and thereby sensitize cells to ionizing radiation. DNA double strand repair was assessed in a cell-free system using PC3 cell lysate, and a linearized pGEM3Z plasmid containing an insert with a BamH1 restriction site flanked by polyamide binding site motifs, and analyzed by qPCR. Rejoining of the linearized plasmid was reduced by $85 \%$ and inhibited rejoining by DNA ligase compared to untreated controls. Neutral comet assay revealed no genomic fragmentation after $48 \mathrm{~h}$ of $10 \mu \mathrm{M}$ polyamide treatment. Genomic fragmentation was statistically similar $1 \mathrm{~h}$ post radiation with $10 \mathrm{~Gy},+/-$ polyamide. However, at $24 \mathrm{~h}$ post radiation, cells pretreated with $10 \mu \mathrm{M}$ polyamide had persistently elevated tail lengths (181 vs. $129 p<0.001)$ and tail moment (103 vs $74 p<0.001)$. After radiation, greater than $95 \%$ of cells $+/-10 \mu \mathrm{M}$ polyamide pretreatment demonstrated increased $\gamma$-H2AX phosphorylation. $24 \mathrm{~h}$ post RT, $32 \%$ of polyamide treated cells continued to have elevated $\gamma$-H2AX phosphorylation, whereas only $19 \%$ of untreated cells continued to have elevated $\gamma$-H2AX phosphorylation. Polyamide treatment with $10 \mu \mathrm{M}$ resulted in a sensitization enhancement ratio of 2.48 at 4 Gy by clonogenic assay. In conclusion Py-Im polyamides are non-genotoxic and sequence specific; potentially offering a strategy for sequence-directed interference with DNA repair and other DNA-depended processes.

\section{Clin-11}

\section{SBRT of the lung: Current practice regarding centrally localized NSCLC within the DEGRO Workgroup of Stereotactic Radiotherapy}

\section{Dr. J. Roesch, Prof. Dr. M. Guckenberger}

\section{UniSpital Zürich, Klinik für Radio-onkologie, Zürich, Switzerland}

Aims This study assesses current clinical practice regarding SBRT of centrally located lung tumors, to identify common fractionation schedules and contraindications.

Methods A questionnaire consisting of two parts was handed to participants of the DEGRO Workgroup of stereotactic radiotherapy, representing centers in Germany and Switzerland. The first part of the questionnaire covers general information about the centers, whereas the second one specifically addresses SBRT of centrally located lung tumors, using the example of nine primary NSCLC cases. Reconstructions of a contrast enhanced CT, as well as PET-Imaging for each case were available to participants.

Results The majority of centers which responded $(n=26)$ were academic $(73 \%)$, provided an interdisciplinary tumorboard $(88 \%)$ and offered SBRT for lung tumors in general $(96 \%)$. Two centers questioned the suitability of SBRT for central lung tumors because of a lack of evidence. Most centers were experienced in SBRT for central lung tumors $(88 \%)$ and half of the centers reported of more than ten treated cases within an average timespan of 6.9 years. As their main fractionation schedule the majority used equivalent eight-fraction schemes of doses per fraction ranging between 6-7.5 Gy applied to the 65-90\%-isodoseline surrounding the PTV.

Indication for SBRT was confirmed by $>76 \%$ of centers in 6 of 9 cases. In these cases no tumor exceeded $5 \mathrm{~cm}$ in diameter or reached less than $3 \mathrm{~cm}$ to the carina. Previous pneumonectomy or adjacent esophagus were no contraindications. One case would have been treated with SBRT by only one center. Here the tumor reached to the carina and its diameter was $7 \mathrm{~cm}$.

Conclusion Within DEGRO Workgroup of stereotactic radiotherapy, early-stage central NSCLC is an indication for SBRT if tumordiameter $<5 \mathrm{~cm}$ and distance to carina $>3 \mathrm{~cm}$.

\section{Clin-12}

Patterns of Failure After Stereotactic Body Radiation Therapy (SBRT) of Primary Lung Tumours and Pulmonary Metastases

P. Rogowski ${ }^{1}$, S. Brodmann ${ }^{1}$, K. Steinauer ${ }^{1}$, T. Hess ${ }^{2}$, U.R. Meier ${ }^{1}$, R. Gräter ${ }^{1}$

${ }^{1}$ Klinik für Radio Onkologie, Kantonsspital Winterthur, Winterthur, Switzerland; ${ }^{2}$ Departement Innere Medizin, Pneumologie, Kantonsspital Winterthur, Winterthur, Switzerland

Aims To determine the patterns of failure in patients with non-small cell lung cancer or pulmonary metastases treated with SBRT.

Methods Retrospective analysis of 39 patients $(\mathrm{f}=15, \mathrm{~m}=24)$, median age 70.7 years $(46.8$ y -86.5 y) with lung cancer $(n=20)$ and pulmonary metastases $(n=19)$, treated with SBRT from $06 / 2011$ to $02 / 2015$. Sixteen of 20 lesions in patients with lung cancer were histologically confirmed. $60 \%(n=12)$ of primary lung cancers were stage T1, 35\% $(n=7)$ were stage T2 and $5 \%(n=1)$ were stage T3 (AJCC 7 th ed). Patients were treated with a median total dose of $60.0 \mathrm{~Gy}$ (30.0-60.0 Gy), which was administered in 3-8 fractions. Endpoints were local control, freedom from metastases and overall survival.

Results After a median follow-up of 1.3 years, local control was $92 \%$ (36/39 patients). 1 patient (with lung cancer) had local recurrence only, 2 of 39 patients (one with lung cancer, one with metastatic disease) developed local recurrence and distant metastases. Those 3 patients are still alive after a median follow-up of 1.4 years. Overall $31 \%(12 / 39)$ of patients developed distant metastases (after a median time of 1.3 years). In the group of patients with primary lung cancer $80 \%(16 / 20)$ were free from metastases. In the group of patients with pulmonary metastases, $58 \%(11 / 19)$ had no further metastatic spread. $44 \%(17 / 39)$ of the patients died during the observation period (after a median time of 2 years). Death rate was $35 \%(7 / 20)$ in the group with primary lung cancers treated with SBRT and $52 \%(10 / 19)$ in the group with pulmonary metastases treated with SBRT.

Conclusion SBRT is an excellent treatment option for patients with non-small cell lung cancer and oligometastatic pulmonary tumours, which are not candidates for surgical resection and results in a high rate of local control.

\section{Clin-13}

\section{Radiotherapy in patients with brain metastasis from solid tumors: Critical appraisal of a single institution analysis}

J.J. Stelmes, N.C. Azinwi, M.C. Valli, K. Yordanov, S. Cima, F. Martucci, S. Presilla, A. Richetti, G. Pesce

Radiation Oncology, Oncology Institute of Southern Switzerland (IOSI), Bellinzona-Lugano, Medical Physics EOC, Bellinzona, Switzerland

Aims To give a critical view of clinical outcome in patients (pts) with brain metastasis based on overall survival (OS) treatment technique (tt) and local control (LC).

Methods Single Institution retrospective analysis of pts treated in the period between january 2013 and march 2015. An estimate of the RPA status was done. Long-term-survival was defined as OS rate superior to 6 months.

Results One-hundred-and-six pts were identified. Mean age was 62 (26-88). The most frequent histology were NSCLC (30\%). About $80 \%$ of the treated pts were in the RPA II class. WBRT was administered in $54 \%$ of the pts, SBRT in $38 \%$, stereotactic RT of the surgical bed (SRTSB) in $17 \%$. The most prescribed dose was 25 Gy in case of SRTSB (44\%) and $40 \mathrm{~Gy}$ in the exclusive SBRT (41\%). The OS was 146 days. Fourteen percent of the pts who underwent WBRT died during the first month with a OS of 115 days. The OS in SRTSB group was 
277 days and in the RT exclusive group 149 days $(p=0.073)$. LC rates at 3 and 6 months in the SBRT and WBRT group were 90 and $93 \%$ respectively 81 and $90 \%$. The OS in the FFF (flattening filter free) group was 150 days and in the non FFF group was 142 days.

Conclusion SBRT as exclusive or adjuvant therapy to the surgical bed was effective in selected pts with better OS compared to the WBRT group..Our data shows a trend for better OS rate in the adjuvant RT group. More than $10 \%$ of the pts in the WBRT group died in the first month. There were no differences in median OS time considering the tt. LC rate were similar in the SBRT and WBRT group. This analysis also confirm the importance of pts allocation to treatments according to validated prognostic factors.

\section{Clin-14}

Does breast size and body mass index influence the reliability of the heart and the left anterior descending coronary artery delineation with automatic segmentation in breast cancer patients simulated in prone position?

X. Wang M.D. ${ }^{1,2}$, V. Chatelain-Fontanella M.D. ${ }^{1}$, J. P. Vallée M.D. ${ }^{3}$ R. Miralbell M.D. ${ }^{1}$, G. Dipasquale M.Sc.

${ }^{1}$ Department of Radiation Oncology, Geneva University Hospital, Geneva, Switzerland; ${ }^{2}$ Department of Radiation Oncology, Tianjin Union Medical Center, Tianjin, China; ${ }^{3}$ Department of Radiology, Geneva University Hospital, Geneva, Switzerland

Aim To evaluate the impact of anatomic patients' (pts) characteristics on the reliability of an atlas-based automatic-segmentation (AS) tool for contouring simultaneously left breast clinical target volume (CTV), heart, and left anterior descending artery (LADA) in patients simulated in prone position.

Methods A library with ten pts was created and contoured (atlas for $\mathrm{AS}$ ) by a senior ( $\mathrm{Sr}$ ) radiation-oncologist. The $\mathrm{Sr}$ also contoured ten additional pts (test cases). We assessed the reliability of AS comparing test and atlas pts using the breast size volume $(<600 \mathrm{cc}, 600-1100 \mathrm{cc}$, $>1100 \mathrm{cc}$ ) vs. cup size (B, C, and D) and body mass index (BMI) (22, 26 , and 30 ). AS was optimized by manually matching on the heart, thus correcting for translations and rotations. To analyze the results a dice index was obtained (for the CTV, heart, and LADA $+1 \mathrm{~cm}$ ) comparing the AS contours with the $\mathrm{Sr}$ ones used as reference.

Results No statistical difference was seen between the two groups for the CTV (mean \pm SD dice index $\approx 0.91 \pm 0.04$ ). The heart mean dice index improved when using the cup size and the BMI, from $0.88 \pm 0.05$ to $0.91 \pm 0.02(p=0.03)$. The LADA $+1 \mathrm{~cm}$ minimum dice index also improved when using the cup size and the BMI (from 0.25 to 0.55 ) though without statistical differences between mean dice values. There was no correlation between the dice of the heart and the LADA $+1 \mathrm{~cm}$ when matching on the heart.

Conclusions In the present study we have been able to show that breast cup size and most of all BMI can help to improve the heart and the LADA delineation with AS.

\section{Physics}

Phys-01

\section{First year of clinical experience with the Gantry 2 @ PSI}

F. Albertini, A. Bolsi, M. De Prado, F. Emert, A. Fredh, F. Gagnon-Moisan, C. Goma, J. Hrbacek, S. König, L. Lederer,

A. Lehde, R. Malyapa, L. Mikroutsikos, D. Meer, D. Oxley,

L. Placidi, S. Safai, D. Scandurra, R. Schneider, P. Thoma,

P. Trnkova, R. van der Meer, A.J. Lomax, D.C. Weber
Center for Proton Radiation Therapy, Paul Scherrer Institut (PSI), Villigen, Switzerland

Aims The first patient was treated in Gantry 2 (G2) in November 2013. G2 is an iso-centric, compact Gantry with a diameter of only $8.5 \mathrm{~m}$, with fast 3D scanning (change in energy $<100 \mathrm{~ms}$ ) and with narrow pencil beams $(3-5 \mathrm{~mm})$.Hereby we report our initial experience.

Methods and material 30 patients have been treated with G2 during the first 18 months of clinical operation with both SFUD and IMPT plans. Absolute dose, beam range and the spot position are measured during the daily QA check procedure for different beam energies and gantry angles.

Patient specific dose verifications are performed before the delivery with a commercial PTW 2D array. Results are analyzed using the gamma $(3 \% / 3 \mathrm{~mm})$ evaluation.

Delivered dose distributions have been reconstructed for selected fraction in the patient geometries using a machine logfile driven independent dose calculation.

Results Fig. 1 shows an example of the plan and DVHs for the first patient treated at G2. In total, 60 treatment series have been applied, with a mean of 3.25 fields delivered per plan. PTV volumes treated are in the range of $20-310 \mathrm{ml}$, with a median treatment time per individual field of $35 \mathrm{~s}$

Results of the daily QA checks of absolute dose and beam range are excellent (differences in dose $<1 \%$ and in range $<0.5 \mathrm{~mm}$ from the reference values). Patient specific field verifications show a very good agreement $(>98 \%$ of points over all fields passing the gamma criteria). Maximum single point differences of $<5 \%$ have been found over all the analyzed series between the planned and the reconstructed delivered doses calculated from the log-files.

Conclusion The introduction of G2 into clinical routine has been smooth and has resulted in excellent reproducibility and delivery accuracy. Although we have deliberately started with a cautious approach in terms of patient load and target location, our current experience indicates the scope for increased patient throughput and indications.
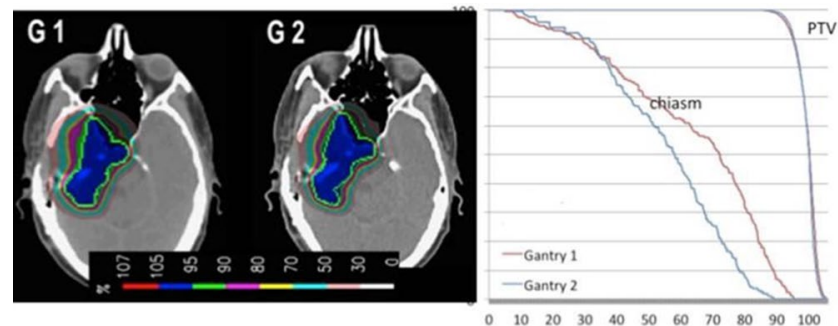

Fig. 1 Phys-01. Dose distribution and DVHs for the very first patient treated in G2 in comparison with the same plan calculated on G1. The better conformality of the plan calculated with the new Gantry is due to the smaller beam size

Phys-02

Evaluation and improvement of proton therapy planning approaches for ependymoma

A. Bolsi, A. Lehde, L. Mikroutsikos, P. Morach, M. De Prado, L. Placidi, R. Malyapa, R. Schneider, F. Albertini, J. Hrbacek, A.J. Lomax, D.C. Weber

Center for Proton Therapy, Paul Scherrer Institute (PSI), Villigen, Switzerland

Aims To assess the effect on the brainstem (BS) dose of various beam arrangements for the treatment of intra-cranial ependymoma with spot-scanning proton therapy. 
Methods This study included 17 children presenting with intra-tentorial ependymoma, treated at PSI between 2011 and 2013. We compared dose distributions, with focus on BS dose as a function of field arrangements per series. We grouped them into three categories: (a) narrow fields (posterior fields separated $<40^{\circ}$ ); (b) non-coplanar fields (couch angle $>20^{\circ}$ for at least 2) and (c) horizontal fields (at least one field close to lateral) Max doses, D2\%, V100\% and V102\% to the BS for each series and for the entire treatment were evaluated. For three sub-selected cases, we retrospectively planned the treatment using: (i) two anterior and two posterior oblique (star); (ii) two lateral and one vertex or one PA; three non-coplanar fields (Figure). Each series was planned with the optimal field arrangement in respect to target coverage and BS dose. Dose metrics for targets and BS were evaluated.

Results Max doses, D2\% and V102\% to the BS were not significantly different among the 3 beam arrangements. Differences were found for $\mathrm{V} 100 \%$, with a $10 \%$ mean decrease for the non-coplanar approach as compared to narrow fields (1st series: from 53 to $43 \%$; 2nd series: from 40 to $29 \%$ ). Results for the horizontal arrangement are in between. V100\% to the BS was also slightly lower if the field arrangement was changed at least once during the treatment (from 14 to $6 \%$ ). The sub-set analysis showed that target coverage and dose to the BS were mainly dependent on the patient's anatomy.

Conclusion We have defined the clinical planning protocol for ependymoma to be composed of different beam arrangements for each series, avoiding the narrow angle.

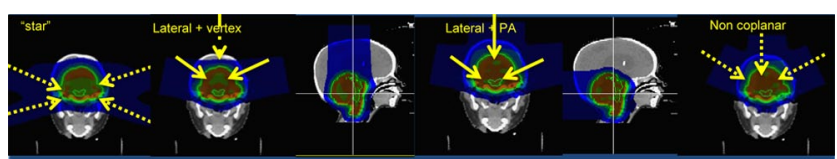

Fig. 1 Phys-02

Phys-03

The effect of anatomical changes on PBS proton dose distributions: a retrospective review of 951 patients treated at PSI

L. Placidi, F. Albertini, A. Bolsi, R. Schneider, R.S. Malyapa, D.C. Weber, A.J. Lomax

Center for Proton Radiation Therapy, Paul Scherrer Institut (PSI), Villigen, Switzerland

Aims During the course of radiotherapy, anatomical changes can affect treatment quality, resulting in time consuming replanning procedures. It is therefore important to know how often these changes occur and what is their potential impact on the delivered dose.

Methods 951 patients treated at PSI between 2000-2014 with PBS proton therapy (PT) were included in this retrospective study. For those where repeat planning CT's were acquired (rePCT), the nominal plan has been recalculated on these and the resulting dosimetric changes assessed using a number of dosimetric parameters.

Results For 244 (25.6\%) patients (Fig. 1) $\geq 1$ control CTs (CCT) have been acquired during the course PT. For $100(9.5 \%)$ of these patients, a rePCT was deemed necessary. For 46 patients, the rePCT was acquired due to anatomical changes occurring during the therapy, with the remaining rePCT's being acquired for other reasons (e.g. new immobilization). For patients treated in the skull base region, maximum dose differences (Dmax) of 8 and $4.6 \%$ were found for the brainstem and chiasma respectively when the original plan was recalculated on the rePCT. For H\&N cases, a Dmax of $19.6 \%$ in the spinal cord was observed for one case. For the pelvis and spinal cord group, Dmax's of $40.6 \%$ in the femoral head and $7 \%$ in the rectum were detected. Maximum reductions of CTV coverage (V95\%) for the skull base, H\&N and spinal cord tumor groups were $10.6,6.6$ and $7.4 \%$ respectively. Conclusion Clinically delivered plans have been found to be robust to anatomical variations with re-planning being deemed necessary in less than $19 \%$ (anatomical rePCT/CCT) of cases. However, as the dosimetric effect of the changes can be large, they have to be monitored and (if detected) evaluated on an individual basis.
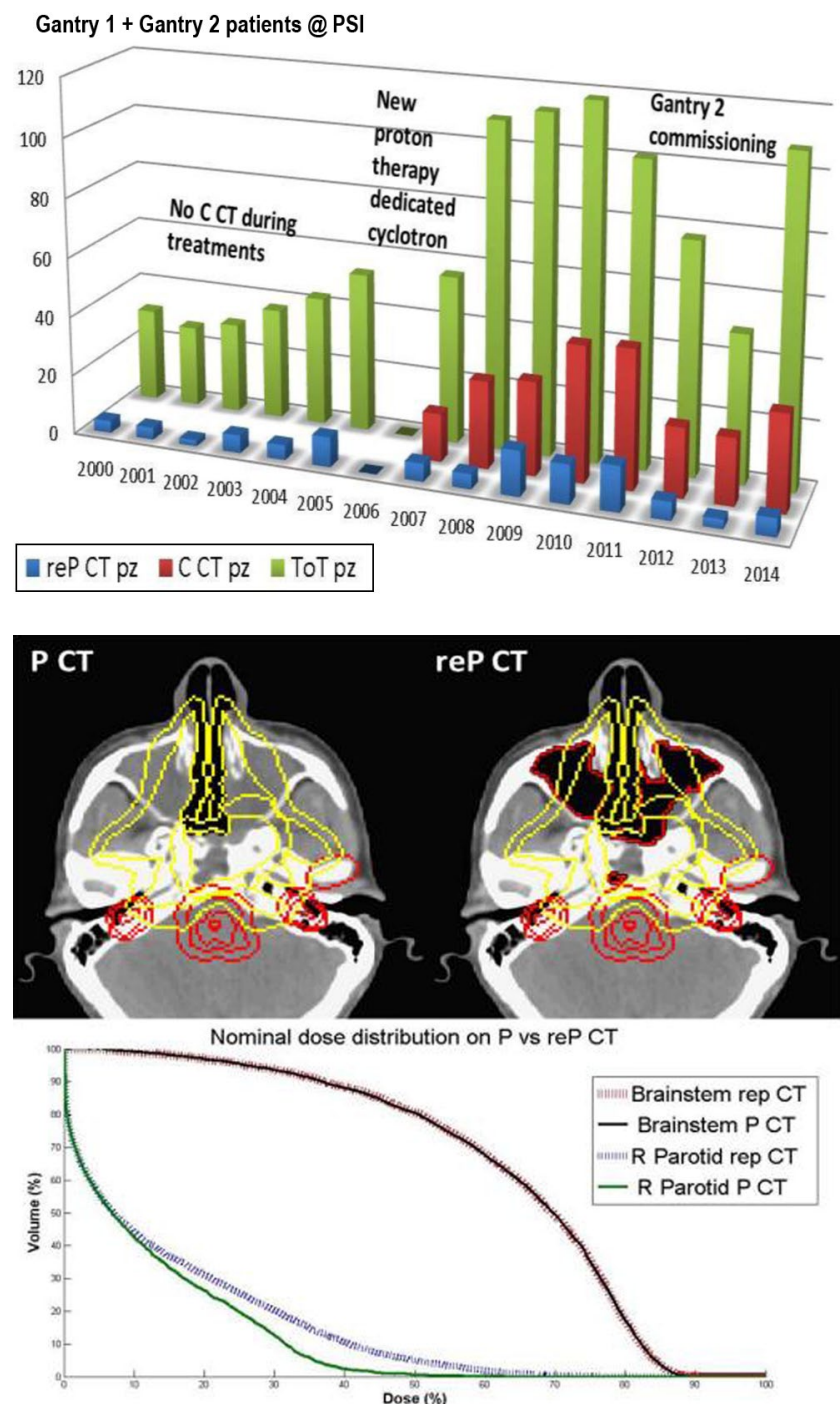

Despite substantial anatomical variations in nasal and paranasal sinusess, none dosimetric effect appears in the brainstem. Visible differences in right parotid need to be clinically evaluated.

Fig. 1 Phys-03

Phys-04

\section{VMAT QA pre treatment using Elekta Iview GT}

\section{Tamburella, G. Guibert, P. Weber}

Service de radiothérapie, Hopital Neuchatelois, La Chaux de Fonds, Switzerland

Aims As the spatial resolution is very high, Electronic Portal Imaging Device is now widely used for quality assurance (QA) of volumetric arc therapy (VMAT) treatments. In this work, we developed a very simple approach to use our portal imager Iview GT-of Elekta Agility 160 for VMAT pre-treatment QA. 
Methods Uniformity and linearity with the dose were first investigated. The conversion from the grey scale to dose (D) was done in the dose range $0.3-2.2 \mathrm{~Gy}$, by an ionisation chamber. A software based on Octave GNU has been developed to correct the matrix of the DICOM images $\mathrm{M}_{\text {iview }}$ into a matrix of dose $\mathrm{M}$ using the formula: $\mathrm{M}=\mathrm{M}_{\text {iview }}{ }^{\text {iview }} \mathrm{D}^{\circledR} \mathrm{M}_{\mathrm{TG}}{ }^{\circledR} \mathrm{M}_{\mathrm{LR}}$.

With $\mathrm{M}_{\mathrm{TG}}$ and $\mathrm{M}_{\mathrm{LR}}$, corrections of reading for Left—Right (LR) and target-Gun (TG) direction respectively.

QA pre treatments for several localisations were checked. The comparison of the iview and the TPS Pinnacle 9.8 from Philips were done by using the $\gamma$ index criteria.

Results 1 . The uniformity showed a difference of reading from the centre to the edges. This difference can reach $6 \%$, and is corrected by two polynomial fits in LR and TG directions.
2. The dose calibration showed a linear correlation between the grey units and the dose. VMAT dosimetries compared to the matrix of dose $\mathrm{M}$ showed a very good correlation according the $\gamma$ index of 3\%-3mm.

These results were (also) compared with those obtained with Octavius 4D from PTW, and did not show major differences.

Conclusion The Ivew GT can be used in routine checks for VMAT pre treatment QA, with good results in relative dosimetry. The results in absolute dosimetry are very promising, but can be improved by adapting the homebuilt software to compensate for the low dose adjustment of the IviewGT. 